Artigo Original

\title{
Efeito da intensidade do exercício de corrida intermitente 30s:15s no tempo de manutenção no ou próximo do $\mathrm{VO}_{2} \max$
}

\author{
Rafael Alves de Aguiar \\ Jardel Schlickmann \\ Tiago Turnes \\ Fabrizio Caputo

\begin{abstract}
Laboratório de Pesquisas em Desempenho Humano, Universidade do Estado de Santa Catarina (UDESC), Florianópolis, SC, Brasil
\end{abstract}

\begin{abstract}
Resumo: O presente estudo comparou o tempo mantido acima de $90 \%\left(\mathrm{t} 90 \mathrm{VO}_{2} \max \right)$ e de $95 \% \mathrm{VO}_{2} \mathrm{max}$ ( $\left(95 \mathrm{VO}_{2} \mathrm{max}\right)$ em três diferentes intensidades de exercício. Após a realização de um teste incremental para determinar $\mathrm{V} \mathrm{VO}_{2} \max$ oito estudantes de educação física ativos ( $23 \pm 3$ anos) executaram três sessões de exercícios intermitentes $\left(100,110\right.$ e $120 \%$ da velocidade do $\mathrm{VO}_{2} \max \left(\mathrm{vVO}_{2} \max \right)$ ) com razão esforço:recuperação de 30s:15s. O t95VO 2 max foi significantemente maior em $110 \% \mathrm{vVO}_{2} \mathrm{max}_{(\mathrm{El}}\left(\mathrm{El}_{110 \%}\right)$ $(218,1 \pm 81,6 \mathrm{~s})$ quando comparado a $100 \% \mathrm{vVO}_{2} \max \left(\mathrm{El}_{100 \%}\right)(91,9 \pm 75,2 \mathrm{~s})$ e a $120 \% \mathrm{vVO}_{2} \max \left(\mathrm{El}_{120 \%}\right)$ $(126,3 \pm 29,4 \mathrm{~s})$, porém sem diferença entre $\mathrm{El}_{100 \%}$ e $\mathrm{El}_{120 \%}$. $\mathrm{O}$ t90 $\mathrm{VO}_{2} \mathrm{max}$ somente apresentou diferença significante entre $\mathrm{El}_{110 \%}$ e $\mathrm{El}_{120 \%}$. Portanto, conclui-se que durante exercício intermitente com razão 30s:15s, a intensidade de $110 \% \mathrm{VVO}_{2}$ max apresenta-se mais adequada para manter o $\mathrm{VO}_{2}$ próximo ou no $\mathrm{VO}_{2} \mathrm{max}$ por um tempo maior.
\end{abstract}

Palavras-chave: Desempenho atlético. Tempo de exaustão. Metabolismo energético.

Effect of intensity of intermittent running exercise $30 \mathrm{~s}: 15 \mathrm{~s}$ at the time maintenance at or near $\mathrm{VO}_{2}$ max

\begin{abstract}
The present study compared the time maintained above $90 \%$ ( $\mathrm{t} 90 \mathrm{VO}_{2} \max$ ) or $95 \% \mathrm{VO}_{2} \max$ $\left(\mathrm{t} 95 \mathrm{VO}_{2} \mathrm{max}\right)$ in three different exercise intensities. After performing an incremental test to determine $\mathrm{VO}_{2} \mathrm{max}$, eight physical education active students $(23 \pm 3$ years) performed three intermittent exercise sessions $\left(100,110\right.$ e $120 \%$ velocity of $\mathrm{VO}_{2} \max \left(\mathrm{vVO}_{2} \max \right)$ ) with ratio effort:recovery of $30 \mathrm{~s}: 15 \mathrm{~s}$. The $\mathrm{t} 95 \% \mathrm{VO}_{2} \max$ was significantly higher at $110 \% \mathrm{VVO}_{2} \max \left(\mathrm{El}_{110 \%}\right)(218.1 \pm 81.6 \mathrm{~s})$ compared to $100 \%$ $\mathrm{VVO}_{2} \max \left(\mathrm{El}_{100 \%}\right)(91.9 \pm 75.2 \mathrm{~s})$ and $120 \% \mathrm{VVO}_{2} \max \left(\mathrm{El}_{120 \%}\right)(126.3 \pm 29.4 \mathrm{~s})$, but without differences between $\mathrm{El}_{100 \%}$ and $\mathrm{El}_{120 \%}$. The $\mathrm{t} 90 \% \mathrm{vVO}_{2}$ max was significantly different only between $\mathrm{El}_{110 \%}$ and $120 \%$. Therefore, we conclude that during intermittent exercise with ratio $30 \mathrm{~s}: 15 \mathrm{~s}$, the intensity of $110 \% \mathrm{vVO} \mathrm{O}_{2} \mathrm{max}$ appears more appropriate to maintain $\mathrm{VO}_{2}$ max for a longer time.
\end{abstract}

Keywords: Athletic performance. Time to exhaustion. Energy metabolism.

\section{Introdução}

O consumo máximo de oxigênio $\left(\mathrm{VO}_{2} \max \right)$ é definido como a maior quantidade de oxigênio que pode ser captada do ar ambiente, transportada e utilizada pelas células durante a atividade física (HILL e LUPTON, 1923; HAWKINS et al., 2007). Dentre as características fisiológicas encontradas em atletas, o $\mathrm{VO}_{2}$ max é um dos mais relevantes para determinar a eficiência do sistema cardiorrespiratório, e um importante indicador do desempenho para atletas de provas com alta demanda do sistema aeróbio, como as corridas de fundo e meio fundo no atletismo (BRANDON, 1995; MIDGLEY, MCNAUGHTON e WILKINSON, 2006; MIDGLEY, MCNAUGHTON e JONES, 2007).

Nesse sentido, parece conveniente encontrar métodos de treinamento que melhorem 0
$\mathrm{VO}_{2}$ max. Assim, alguns pesquisadores têm assumido que o treino mais efetivo para modificar esta variável, seria aquele que estimula o indivíduo a manter o maior tempo possível próximo ou na intensidade do $\mathrm{VO}_{2} \mathrm{max}$ (ROBINSON et al., 1991; MIDGLEY e MC NAUGHTON, 2006). Este tipo de treinamento pode sobrecarregar ao máximo as estruturas orgânicas que limitam o $\mathrm{VO}_{2} \max$, proporcionando um estímulo ideal para a adaptação fisiológica (MIDGLEY e MC NAUGHTON, 2006). Tem sido assumido que o tempo mantido em altos percentuais do $\mathrm{VO}_{2} \max$, acima de $90 \%$ $\left(\mathrm{t} 90 \mathrm{VO}_{2} \max \right)$ ou $95 \%$ do $\mathrm{VO}_{2} \max \left(\mathrm{t}^{2} 5 \mathrm{VO}_{2} \max \right)$ (DUPONT et al., 2002; MIDGLEY e MC NAUGHTON, 2006), poderia ser usado como um critério relevante para caracterizar e analisar o estímulo do exercício. 
Alguns estudos preconizam que o aumento na intensidade do treinamento é a principal forma de conseguimos adaptações no $\mathrm{VO}_{2} \max (\mathrm{FOX}$ et al., 1973; WENGER e BELL, 1986; IAIA e BANGSBO, 2010). Deste modo, o exercício intermitente (EI), que é caracterizado por períodos de esforço intercalados por períodos de recuperação, pode ser uma excelente forma para intensificar os treinamentos e melhorar a potência aeróbia (DE LUCAS, DENADAI e GRECO, 2009). Intensidades supramáximas (i.e. acima da velocidade do $\left.\mathrm{VO}_{2} \max \left(\mathrm{VVO}_{2} \max \right)\right)$ podem ser mais adequadas durante El com baixa duração de esforço ( $\leq 30 \mathrm{~s}$ ), visto que intensidades menores podem manter pouco tempo próximo ou no $\mathrm{VO}_{2} \max$, ou ainda ser insuficiente para alcançá-lo (GOROSTIAGA et al., 1991; MILLET, CANDAU et al., 2003).

Baseado nos pressupostos acima, diferentes estudos analisaram o efeito da intensidade do exercício intermitente no $\mathrm{tVO}_{2}$ max utilizando uma razão esforço:recuperação de $1: 1$, sendo $30 \mathrm{~s}: 30 \mathrm{~s}$ (BILLAT, SLAWINSKI et al., 2000; MILLET, LIBICZ et al., 2003; THEVENET et al., 2008), ou 15s:15s (BILLAT et al., 2001; DUPONT et al., 2002). Contudo, a razão esforço:recuperação de 2:1 apresentou melhores resultados para t95 $\mathrm{VO}_{2} \max$ do que exercícios com razão esforço:pausa de 1:1 (MILLET, CANDAU et al., 2003). Assim, a utilização de 30s de esforço intercalados por $15 \mathrm{~s}$ de recuperação, pode ser um ótimo estímulo para aumentar o tempo mantido em altos percentuais do $\mathrm{VO}_{2} \max$, visto que, $30 \mathrm{~s}$ de esforço é suficiente para o $\mathrm{VO}_{2}$ alcançar o seu valor máximo após algumas séries de exercício (THEVENET et al., 2007). Além disso, um menor tempo de recuperação (15s) permite manter o $\mathrm{VO}_{2}$ ainda elevado ao final da recuperação, fazendo com que o tempo para alcançar novamente $\mathrm{O} \mathrm{VO}_{2} \max$ seja reduzido durante a série subseqüente (DUPONT et al., 2002).

Portanto, este estudo tem como objetivo verificar o efeito de três diferentes intensidades de exercício $\left(100,110\right.$ e $120 \%$ da $\left.\mathrm{VVO}_{2} \max \right)$ sobre o $\mathrm{t} 0 \mathrm{VVO}_{2} \mathrm{max}$ e $\mathrm{t} 5 \mathrm{VO}_{2} \mathrm{max}$ durante exercício de corrida intermitente com esforço:recuperação de 30s:15s.

\section{Sujeitos}

\section{Métodos}

Participaram deste estudo oito estudantes de educação física ( $23 \pm 3$ anos; 79,8 \pm 9,3 kg e 176 $\pm 5 \mathrm{~cm}$ ) praticantes de atividade física no mínimo duas vezes por semana. Os sujeitos não possuíam nenhum tipo de lesão, não fumavam nem faziam uso regular de qualquer medicamento. Todos os sujeitos foram informados sobre os objetivos, procedimentos da pesquisa e dos possíveis riscos envolvidos no estudo e logo após assinaram o termo de consentimento livre e esclarecido, concordando em participar voluntariamente da pesquisa. $O$ estudo foi autorizado pelo Comitê de Ética em Pesquisas com Seres Humanos da instituição na qual a pesquisa foi realizada sob o $n^{\circ}$ 100/2010.

\section{Delineamento experimental}

Cada sujeito visitou o laboratório em quatro ocasiões, com intervalo de no mínimo $48 \mathrm{~h}$ entre as seções, finalizando o período de coleta em no máximo duas semanas. Todos os testes foram executados em uma esteira motorizada (INBRAMED Millenium Super Atl, Porto Alegre, Brasil) com $1 \%$ de inclinação. $O \quad \mathrm{VO}_{2}$ foi mensurado respiração a respiração durante todos os testes a partir do gás expirado (Quark PFTergo - Cosmed Srl, Roma, Itália). O analisador de gases foi calibrado antes de cada teste usando o ar ambiente e concentrações conhecidas dos gases $\mathrm{O}_{2}(16 \%)$ e $\mathrm{CO}_{2}(5 \%)$ de acordo com as instruções do fabricante. A turbina do analisador foi calibrada por meio de uma seringa com volume de três litros. Em todas as visitas, amostras de $25 \mu \mathrm{L}$ de sangue capilar do lóbulo da orelha foram coletadas imediatamente, 3 e $5 \mathrm{~min}$ após o término dos testes para determinar as máximas concentrações de lactato sanguíneo ([Lac $\left.]_{\text {pico }}\right)$. Nos casos em que a concentração de lactato sanguíneo no quinto minuto após o exercício foi maior que o terceiro minuto, uma amostra adicional no sétimo minuto após o exercício foi coletada para confirmação do $[\mathrm{Lac}]_{\text {pico. }}$ As amostras de sangue foram imediatamente analisadas usando o aparelho YSI 1500 SELECT (Yellow Springs, Ohio, EUA). Antes de cada teste, o analisador de lactato foi calibrado com uma solução padrão de lactato $(5 \mathrm{mM})$.

\section{Visita 1 - Determinação do $\mathrm{VO}_{2} \max e$ $\mathrm{VVO}_{2}$ max}

$\mathrm{O} \mathrm{VO}_{2} \max$ e a $\mathrm{vVO}_{2}$ max foram determinados a partir de um teste incremental em esteira rolante. A velocidade inicial da esteira foi fixada em $8 \mathrm{~km} . \mathrm{h}-1$ e aumentada em 0,5km.h-1 a cada minuto, até que $\mathrm{o}$ sujeito encerrasse $\mathrm{o}$ teste devido à exaustão voluntária. Os dados do $\mathrm{VO}_{2}$ (respiração a respiração) foram reduzidos à 
média de $15 \mathrm{~s}$, sendo que o maior valor foi considerado como $\circ \mathrm{VO}_{2} \max$. Como critérios para a determinação do $\mathrm{VO}_{2} \max$ foram utilizados $\mathrm{O}$ aparecimento de um estado estável do $\mathrm{VO}_{2}$ apesar de um aumento na velocidade (aumento no $\mathrm{VO}_{2}<150 \mathrm{ml} \mathrm{min}^{-1}$ ) ou quando dois ou mais dos seguintes critérios foram observados: (1) quociente respiratório maior que 1,1 ; (2) visível exaustão; (3) freqüência cardíaca ao final do teste dentro de $10 \mathrm{bpm}$ do valor máximo predito $(220$ idade); e (4) concentração de lactato sanguíneo ao final do exercício maior que $8 \mathrm{mmol}^{-\mathrm{I}^{-1}}$. A $\mathrm{VVO}_{2}$ max foi a velocidade mínima em que o $\mathrm{VO}_{2} \mathrm{max}$ foi atingido (BILLAT et al., 1994). Nos casos em que o sujeito interrompeu o teste antes de finalizar um estágio do protocolo, a velocidade final foi corrigida por meio da fração do tempo gasto no estágio até $\mathrm{o} \mathrm{VO}_{2} \max$ ser alcançado multiplicado por $0,5 \mathrm{~km} \cdot \mathrm{h}^{-1}$.

Visitas 2,3 e 4 - Corrida intermitente a 100, 110 e $120 \%$ da $\mathrm{VVO}_{2} \max$

Nas visitas 2, 3 e 4 , após $10 \mathrm{~min}$ de aquecimento a $65 \% \mathrm{vVO}_{2}$ max e $5 \mathrm{~min}$ de repouso passivo, os sujeitos executaram três sessões de exercícios intermitentes de 30 s de corrida a $100 \%$ $\mathrm{vVO}_{2} \max \left(\mathrm{El}_{100 \%)}, 110 \% \mathrm{vVO}_{2} \max \left(\mathrm{El}_{110 \%}\right.\right.$ e $120 \%$ $\mathrm{VVO}_{2} \max \left(\mathrm{El}_{120 \%}\right)$, alternados com $15 \mathrm{~s}$ de recuperação passiva. Todos os testes foram realizados até a exaustão voluntária e em ordem aleatória. Para entrada e saída da esteira, o sujeito se apoiava segurando nas barras laterais $\mathrm{e}$ posicionava seus pés fora do tapete da esteira. $O$ tempo de exaustão (Tlim) foi determinado como o tempo em que o sujeito executou a corrida, não incluindo o tempo de recuperação. A distância percorrida (Dlim) foi determinada como a distância total realizada durante o exercício intermitente. $\mathrm{O} \mathrm{VO}_{2}$ durante o exercício foi fixado em valores médios de $5 \mathrm{~s}$, assim, o $\mathrm{t}^{2} \mathrm{VVO}_{2} \mathrm{max}$ e o $\mathrm{t} 0 \mathrm{VO}_{2} \mathrm{max}$ foram determinados como a somatória de tempo durante 0 protocolo intermitente em que o $\mathrm{VO}_{2}>95 \%$ do $\mathrm{VO}_{2}$ max e o $\mathrm{VO}_{2}>90 \%$ do $\mathrm{VO}_{2} \max$ do teste incremental, respectivamente. Além disso, o $\mathrm{VO}_{2 \text { pico }}$ foi determinado como a maior média de dois valores consecutivos de $5 \mathrm{~s}$.

\section{Análise estatística}

Os dados foram expressos como média \pm DP. A normalidade das variáveis determinadas neste estudo foi analisada pelo teste de Shapiro Wilk. A análise de variância ANOVA one-way, complementada pelo teste de Tukey, foi utilizada para comparar $\mathrm{O} \mathrm{VO}_{2} \mathrm{max}$ e a [lac $]_{\text {pico }}$ do teste incremental com $\circ \mathrm{VO}_{2 \text { pico }} \mathrm{e}$ a $[\mathrm{lac}]_{\text {pico }}$ nas diferentes sessões de exercício intermitente. Além disso, foi utilizada para comparar o Tlim, Dlim, t90 $\mathrm{VO}_{2} \max$ e $\mathrm{t} 5 \mathrm{VOO}_{2} \mathrm{max}$ entre as diferentes sessões de exercício intermitente. $O$ teste de correlação de Pearson foi utilizado para verificar a associação entre as variáveis. Em todos os testes foi adotado um nível de significância de $p<0,05$.

\section{Resultados}

\section{Teste incremental}

Seis dos oito sujeitos apresentaram um estado estável no $\mathrm{VO}_{2}$ e os outros dois sujeitos apresentaram três dos critérios mencionados acima. Os valores médios do $\mathrm{VO}_{2} \max$ e [lac $]_{\text {pico }}$ do teste incremental estão descritos na tabela 1 . Os valores médios da $\mathrm{VVO}_{2} \mathrm{max}$, tempo de exaustão e freqüência cardíaca máxima do teste incremental foram: $14,3 \pm 1,2 \mathrm{~km} \cdot \mathrm{h}^{-1}, 13,5 \pm 2,2$ min e $192 \pm 5 \mathrm{bpm}$, respectivamente.

\section{Variáveis do exercício intermitente}

$\mathrm{Na}$ tabela 1 apresentam-se os dados obtidos a partir dos três testes de corrida intermitente. Não ocorreu diferença no $\mathrm{VO}_{2}$ pico entre os três exercícios intermitentes. Além disso, estes não foram diferentes do $\mathrm{VO}_{2} \max$ obtido no teste incremental $(p=0,144)$. Em El $\mathrm{I}_{100 \%}$, o tempo total, tempo de exercício, quantidade de séries executadas e distância percorrida foram significantemente maiores que em $\mathrm{El}_{110 \%}$ e $\mathrm{El}_{120 \%}$ $(p<0,001)$. Ainda, estas variáveis foram significantemente maiores em $\mathrm{El}_{110 \%}$ quando comparadas a $\mathrm{El}_{120 \%}(\mathrm{p}<0,001)$.

\section{$\mathrm{t} \mathrm{OVO} \mathrm{V}_{2} \max$ e $\mathrm{t} 95 \mathrm{VO}_{2} \max$}

$\mathrm{Na}$ Figura 1 é demonstrado o tempo mantido acima de $90 \%\left(\mathrm{t} 0 \mathrm{VO}_{2} \max \right)$ e $95 \%\left(\mathrm{t}^{2} 5 \mathrm{VO}_{2} \max \right)$ do $\mathrm{VO}_{2} \mathrm{max}$ em valores absolutos e relativos ao Tlim a 100, 110 e $120 \%$ da $v \mathrm{VO}_{2} \max$. Diferenças significantes foram encontradas no $\mathrm{t}^{2} 5 \mathrm{VO}_{2} \mathrm{max}$ entre $\mathrm{El}_{110 \%}$ e $\mathrm{El}_{100 \%}(\mathrm{p}=0,01), \mathrm{El}_{110 \%}$ e $\mathrm{El}_{120 \%}(\mathrm{p}<$ $0,05)$, porém não entre $\mathrm{El}_{120 \%}$ e $\mathrm{El}_{100 \%}(\mathrm{p}=0,6)$. Quando $\mathrm{t} 5 \mathrm{VO}_{2} \mathrm{max}$ em valores relativos foi analisado, $\mathrm{El}_{120 \%}$ e $\mathrm{El}_{110 \%}$ apresentaram valores significantemente maiores quando comparado a $\mathrm{EI}_{100 \%}(\mathrm{p}<0,001)$, entretanto sem diferenças entre as duas intensidades $(p=0,08)$. Em relação ao valor absoluto de $\mathrm{t} 0 \mathrm{VO}_{2} \mathrm{max}, \mathrm{El}_{110 \%}$ foi significantemente maior quando comparado a $E_{120 \%} \quad(p<0,05)$, entretanto, $E I_{100 \%}$ não apresentou diferença em relação a $\mathrm{El}_{110 \%}(\mathrm{p}=$ 
$0,51)$ e $\mathrm{El}_{120 \%}(\mathrm{p}=0,15)$. Quando o t90VO $\max$ foi analisado em percentual do Tlim, $\mathrm{El}_{120 \%}$ e $\mathrm{El}_{110 \%}$ foram significantemente maiores que $\mathrm{El}_{100 \%}(\mathrm{p}<$
0,001), sem diferenças entre $\mathrm{El}_{120 \%}$ e $\mathrm{El}_{110 \%}$ ( $\mathrm{p}=$ 0,97). A Figura 2 apresenta um exemplo da resposta do $\mathrm{VO}_{2}$ durante $\mathrm{El}_{100 \%}, \mathrm{El}_{110 \%}$ e $\mathrm{El}_{120 \%}$.

Tabela 1. Dados referentes aos Exercícios Intermitentes. Valores em média \pm DP. Tempo total (tempo de exercício + tempo de recuperação). ${ }^{*}$ Valores referentes a sete sujeitos. ${ }^{a}$ Diferença significante em relação a $100 \%$, ${ }^{b}$ Diferença significante em relação a $110 \%(p<0,05)$.

\begin{tabular}{lcccc}
\hline & Incremental & $\mathrm{El}_{100 \%}$ & $\mathrm{El}_{110 \%}$ & $\mathrm{El}_{120 \%}$ \\
\hline $\mathrm{VO}_{2 \text { pico }}\left(\mathrm{ml} \cdot \mathrm{kg}^{-1} \cdot \mathrm{min}^{-1}\right)$ & $49,7 \pm 3,7$ & $49,0 \pm 2,9$ & $51,7 \pm 3,1$ & $51,1 \pm 5,3$ \\
Tempo total (s) & & $1493 \pm 212$ & $717 \pm 126^{\mathrm{a}}$ & $333 \pm 45^{\mathrm{ab}}$ \\
Tempo de exercício (s) & & $1021 \pm 143$ & $490 \pm 79^{\mathrm{a}}$ & $231 \pm 29^{\mathrm{ab}}$ \\
Séries executadas & & $16 \pm 3^{\mathrm{a}}$ & $8 \pm 1^{\mathrm{ab}}$ \\
Distância percorrida (m) & & $3997 \pm 734$ & $2135 \pm 433^{\mathrm{a}}$ & $1093 \pm 111^{\mathrm{ab}}$ \\
[Lac]pico $\left(\mathrm{mmol.I^{-1 }}\right)^{*}$ & $8,4 \pm 3,1^{\mathrm{a}}$ & $6,1 \pm 1,7$ & $7,7 \pm 2,5^{\mathrm{a}}$ & $9,5 \pm 2,2^{\mathrm{ab}}$ \\
\hline
\end{tabular}
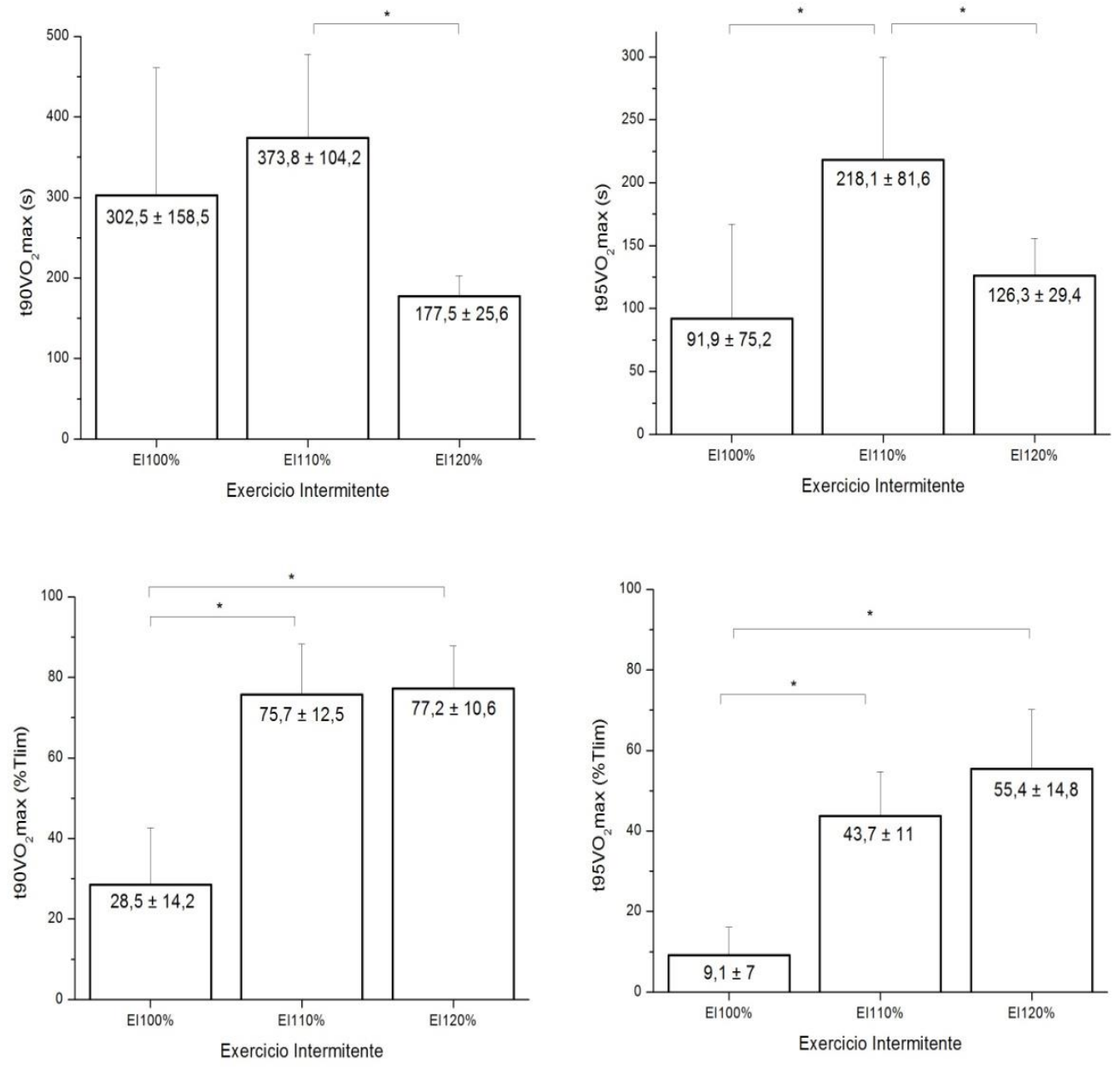

Figura 1. Tempo mantido acima de $90 \%$ e $95 \%$ do $\mathrm{VO}_{2}$ max em valores absolutos e relativos ao Tlim a 100 , 110 e $120 \%$ da $\mathrm{VVO}_{2} \max$. As colunas representam a média com as barras verticais representando um DP. ${ }^{*}$ Diferença significante entre as intensidades $(p<0,05)$.

\section{Discussão}

O propósito deste estudo foi analisar o efeito de três diferentes intensidades de exercício (100, 110 e $120 \%$ da $\mathrm{VVO}_{2} \max$ ) sobre o tempo mantido acima de $90 \%$ ou $95 \%$ do $\mathrm{VO}_{2} \max$ durante exercício intermitente com esforço:recuperação de 30s:15s. Os resultados revelaram que durante $\mathrm{El}_{110 \%} \circ \mathrm{VO}_{2}$ permaneceu por um tempo maior 
sendo mantido em percentuais próximos ao $\mathrm{VO}_{2}$ max. Portanto, dentre as intensidades analisadas, $110 \% \mathrm{vVO}_{2}$ max no exercício realizado com esforço:recuperação de 30s:15s parece ser a melhor intensidade durante sessões de treinamento intermitente no qual o objetivo é aumentar $\circ \mathrm{VO}_{2} \max$.

Alguns estudos têm sugerido que a utilização de uma recuperação passiva durante exercícios intermitentes poderia dificultar 0 alcance do $\mathrm{VO}_{2} \max$ (GOROSTIAGA et al., 1991; TURNER et al., 2006). Entretanto, este aspecto somente foi analisado em exercícios intermitentes com taxa de esforço:recuperação $\leq 1: 1$ e com tempo de exercício $\leq 30$ s. No presente trabalho, o exercício intermitente com taxa de esforço:recuperação de 2:1 e tempo de exercício igual a 30 s foi suficiente para que $\circ \mathrm{VO}_{2}$ alcançasse $\circ \mathrm{VO}_{2} \max$ do teste incremental nas três intensidades analisadas $\left(100,110\right.$ e $120 \%$ da $\left.\mathrm{vVO}_{2} \max \right)$. No entanto, mesmo as três intensidades sendo suficientes para alcançar $\circ \mathrm{VO}_{2} \mathrm{max}$, elas apresentaram diferenças no tempo de manutenção em altos percentuais do $\mathrm{VO}_{2} \max$ (i.e. acima de $90 \% \mathrm{VO}_{2}$ max), fato este possivelmente relacionado à flutuação do consumo de oxigênio durante os períodos de esforço e recuperação, bem como diferenças no Tlim.
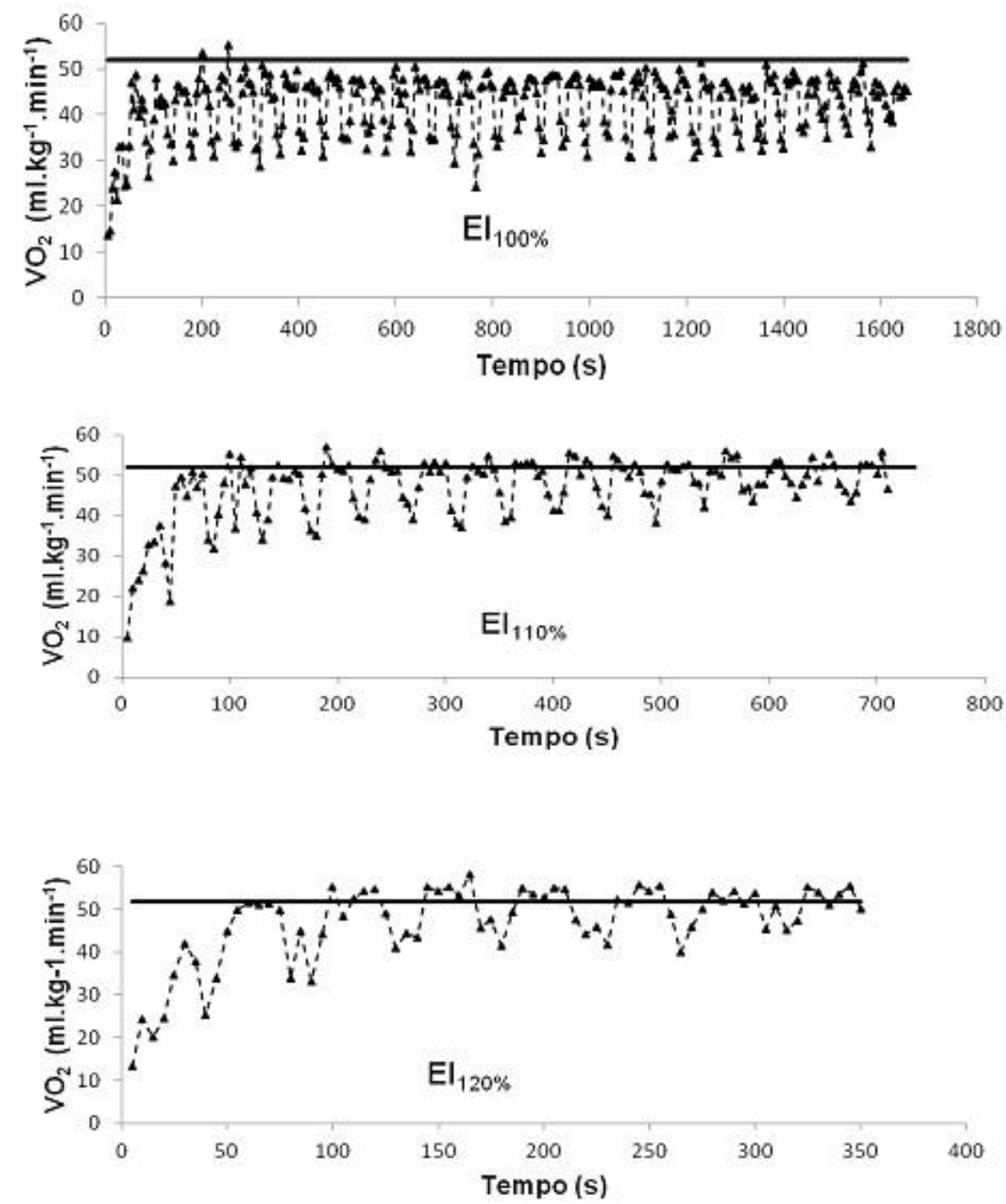

Figura 2. Um exemplo do $\mathrm{VO}_{2}$ pelo tempo no $\mathrm{El}_{100 \%}, \mathrm{El}_{110 \%}$ e $\mathrm{El}_{120 \%}$ para um sujeito representativo. Cada ponto corresponde a média de $5 \mathrm{~s}$. A linha sólida indica o valor de $\mathrm{VO}_{2}$ max determinado durante o teste incremental.

Durante o El de alta intensidade $\left(\geq \mathrm{vVO}_{2} \mathrm{max}\right)$, a energia para execução do exercício é gerada pelo metabolismo anaeróbio, derivada principalmente da creatina fosfato (CF) (SPRIET, HOWLETT e HEIGENHAUSER, 2000), e pelo metabolismo aeróbio, através dos estoques de $\mathrm{O}_{2}$ ligados a mioglobina (ESSEN, HAGENFELDT e KAIJSER, 1977). Portanto, o $\mathrm{VO}_{2}$ durante os períodos de recuperação estão intimamente ligados a reposição parcial desses estoques (BORSHEIM e BAHR, 2003). Spriet et al. (2000) observaram que quanto maior a intensidade do exercício, maior a quebra dos estoques de CF, 
assim, é possível que este aspecto esteja relacionado ao maior $\mathrm{VO}_{2}$ na recuperação em intensidades mais elevadas (BORSHEIM e BAHR, 2003). O comportamento do $\mathrm{VO}_{2}$ durante a recuperação estaria associado a uma maior ressíntese da CF e na restauração dos estoques de $\mathrm{O}_{2}$ da mioglobina, que possivelmente são mais utilizados em intensidades mais elevadas (Figura 2). Além disso, é importante destacar uma progressiva aceleração na cinética do $\mathrm{VO}_{2} \mathrm{com} \mathrm{o}$ aumento da intensidade do exercício contínuo em indivíduos ativos (BILLAT, MORTON et al., 2000; HILL, POOLE e SMITH, 2002), como também uma aceleração ocasionada pelo exercício prévio (BAILEY et al., 2009). Os aspectos citados acima são demonstrados nos valores relativos de t95 $\mathrm{VO}_{2} \max$ e $\mathrm{t} \mathrm{O} \mathrm{VO}_{2} \mathrm{max}$, visto que, foram encontrados maiores percentuais relativos em $\mathrm{El}_{120 \%}$ e $\mathrm{El}_{110 \%}$ em relação a $\mathrm{El}_{100 \%}$, entretanto, comparando $\mathrm{El}_{120 \%}$ e $\mathrm{El}_{110 \%}$, apenas $\mathrm{t}^{2} \% \mathrm{VO}_{2} \max$ apresentou uma tendência de ser maior em $\mathrm{El}_{120 \%}$ $(p<0,08)$.

Diferenças no Tlim também explicam os valores absolutos de tempo mantido em altos percentuais do $\mathrm{VO}_{2} \max$. Nossos resultados demonstraram um efeito da intensidade no Tlim (ver tabela 1), corroborando com outros estudos, que também encontraram um maior tempo de exercício e quantidade de séries executadas quando a intensidade era relativamente menor (DUPONT et al., 2002; THEVENET et al., 2007). Esta resposta poderia ser explicada pela menor contribuição do sistema anaeróbio na ressíntese do ATP (ROZENEK et al., 2007), o que provavelmente diminuiria a produção e acúmulo de metabólitos responsáveis pela instalação da fadiga e conseqüente interrupção do exercício (ver tabela 1). Portanto, para manter mais tempo em $\mathrm{t} 5 \mathrm{VO}_{2} \mathrm{max}$, é necessário uma intensidade intermediária $\left(110 \% \mathrm{vVO}_{2} \max \right)$ no qual alcance o $\mathrm{VO}_{2} \mathrm{max}$ em um tempo reduzido e ainda consiga executar em torno de 15 séries de esforço. Entretanto, para alcançar valores um pouco inferior de $\mathrm{VO}_{2} \max \left(\mathrm{t} 90 \mathrm{VO}_{2} \max \right), \quad \mathrm{El}_{100 \%}$ conseguiu estimular de forma semelhante.

O exercício intermitente utilizado em nosso estudo (esforço:recuperação de 30s:15s) apresentou valores inferiores de tempo mantido em altos percentuais de $\mathrm{VO}_{2}$ max comparado com os valores reportados por Billat et al. (2000), Billat et al. (2001) e Demarie, Koralsztein e Billat (2000), que empregaram El com esforço/recuperação de 30s:30s, 15s:15s e 1/2tlim:1/4tlim (utilização do Tlim na $v^{2} O_{2}$ max), respectivamente. Esta diferença pode ser parcialmente explicada pelas características dos participantes. O presente estudo utilizou estudantes de educação física, enquanto nos outros estudos os participantes eram corredores, portanto, é provável que a cinética mais lenta do $\mathrm{VO}_{2}$ em nossa amostra (CAPUTO e DENADAI, 2004), reduziu o tempo mantido em altos percentuais de $\mathrm{VO}_{2} \max$ (MILLET, LIBICZ et al., 2003).

Em nosso trabalho, utilizamos dois critérios para analisar o tempo mantido em altos percentuais do $\mathrm{VO}_{2} \max \quad\left(\mathrm{t} 0 \mathrm{VO}_{2} \max \mathrm{e}\right.$ t95 $\mathrm{VO}_{2} \mathrm{max}$ ), diferente de outros estudos, que utilizaram somente um dos critérios mencionados acima (MILLET, CANDAU et al., 2003; MILLET, LIBICZ et al., 2003; DUPONT e BERTHOIN, 2004; THEVENET et al., 2008) ou ainda, assumiram como critério $\circ \mathrm{VO}_{2} \max$ menos 2,1 $\mathrm{ml} . \mathrm{kg}^{-1} \cdot \mathrm{min}^{-1}$ (BILLAT, SLAWINSKI et al., 2000). Além do critério adotado, outros fatores podem contribuir para diferenças significantes no tempo mantido em altos percentuais de $\mathrm{VO}_{2}$ max. Midgley, MCnaughton, Carrol. (2007) observaram que a determinação do $t 95 \mathrm{VO}_{2} \max$ pode ser significantemente afetada pelo teste incremental utilizado e pela média utilizada para reduzir os dados de $\mathrm{VO}_{2}$ obtidos durante o exercício. Ainda, é importante destacar que Katch, Sady e Freedson (1982) reportaram uma variação biológica nos valores individuais de $\mathrm{VO}_{2} \max$ de $\pm 5,6 \%$. Desta forma, exige-se cautela na comparação entre as diferentes pesquisas, visto que diferentes critérios foram usados para determinar o tempo mantido em altos percentuais do $\mathrm{VO}_{2} \max$.

Além dos benefícios aeróbios, como maximizar a melhoria no $\mathrm{VO}_{2} \max$ (MIDGLEY e MC NAUGHTON, 2006) e induzir melhoras significantes na densidade mitocondrial (MACDOUGALL et al., 1998), o El com velocidades que induzam uma alta produção de lactato também irão estimular a sua remoção nos intervalos de recuperação. Noakes, Myburgh e Schall (1990), têm destacado que um dos fatores limitantes para as corridas de longa distância seriam as adaptações musculares, portanto, os benefícios do treinamento também dependem da distância percorrida a uma alta velocidade, maximizando o número de contrações musculares potentes. Para estes efeitos, $\mathrm{El}_{100 \%}$ pode ser preferível em relação aos outros El, visto 
que apresentou valores inferiores de lactato sanguíneo ao final do teste e percorreu uma maior distância. Portanto, a escolha da intensidade de exercício durante a sessão de treinamento deve depender dos objetivos da mesma.

Conclui-se que, durante o exercício intermitente com esforço:recuperação de 30s:15s, intensidades entre 100 a $120 \%$ da $\mathrm{vVO}_{2} \max$ induzem uma ampla solicitação do sistema aeróbio. Entretanto, se o objetivo da sessão de treinamento é aumentar o $\mathrm{VO}_{2} \mathrm{max}$, a intensidade equivalente a $110 \%$ da $\mathrm{VVO}_{2}$ max poderia ser a mais efetiva por manter o exercício por um tempo mais prolongado em percentuais próximos ao $\mathrm{VO}_{2} \max$, quando comparada às demais intervenções.

\section{Referências}

BAILEY, S. J.; VANHATALO, A.; WILKERSON, D. P.; DIMENNA, F. J.; JONES, A. M. Optimizing the "priming" effect: influence of prior exercise intensity and recovery duration on $\mathrm{O} 2$ uptake kinetics and severe-intensity exercise tolerance. Journal of Applied Physiology, Bethesda, v. 107, n. 6, p. 1743-1756, 2009. Disponível em: http://www.ncbi.nlm.nih.gov/entrez/query.fcgi?cmd $=$ Retrieve\&db=PubMed\&dopt=Citation\&list uids $=1$ $\underline{9797685}$. Acesso em 10 de fevereiro de 2010

BILLAT, V.; RENOUX, J. C.; PINOTEAU, J.; PETIT, B.; KORALSZTEIN, J. P. Times to exhaustion at $100 \%$ of velocity at VO2max and modelling of the time-limit/velocity relationship in elite long-distance runners. European Journal of Applied Physiology and Occupational Physiology, Berlin, v. 69, n. 3, p. 271-273, 1994. Disponível em:

http://www.ncbi.nlm.nih.gov/entrez/query.fcgi?cmd $=$ Retrieve \&db=PubMed\&dopt $=$ Citation\&list uids $=8$ $\underline{001542}$. Acesso em 12 de janeiro de 2010

BILLAT, V. L.; MORTON, R. H.; BLONDEL, N.; BERTHOIN, S.; BOCQUET, V.; KORALSZTEIN, J. P.; BARSTOW, T. J. Oxygen kinetics and modelling of time to exhaustion whilst running at various velocities at maximal oxygen uptake.

European Journal of Applied Physiology, Berlin, v. 82, n. 3, p. 178-187, 2000. Disponível em:

http://www.ncbi.nlm.nih.gov/entrez/query.fcgi?cmd =Retrieve\&db=PubMed\&dopt=Citation\&list uids $=1$ 0929211. Acesso em 7 de março de 2010

BILLAT, V. L.; SLAWINKSI, J.; BOCQUET, V.; CHASSAING, P.; DEMARLE, A.; KORALSZTEIN, J. P. Very short (15s-15s) interval-training around the critical velocity allows middle-aged runners to maintain VO2 max for 14 minutes. International journal of sports medicine, Stuttgart, v. 22, n. 3, p. 201-208, 2001. Disponível em:

http://www.ncbi.nlm.nih.gov/entrez/query.fcgi?cmd $=$ Retrieve \&db=PubMed\&dopt=Citation\&list uids $=1$ 1354523. Acesso em 15 de janeiro de 2010

BILLAT, V. L.; SLAWINSKI, J.; BOCQUET, V.; DEMARLE, A.; LAFITTE, L.; CHASSAING, P.; KORALSZTEIN, J. P. Intermittent runs at the velocity associated with maximal oxygen uptake enables subjects to remain at maximal oxygen uptake for a longer time than intense but submaximal runs. European Journal of Applied Physiology, Berlin, v. 81, n. 3, p. 188-196, 2000. Disponível em:

http://www.ncbi.nlm.nih.gov/entrez/query.fcgi?cmd $=$ Retrieve\&db=PubMed\&dopt=Citation\&list uids $=1$ $\underline{0638376}$. Acesso em 25 de janeiro de 2010

BORSHEIM, E.; BAHR, R. Effect of exercise intensity, duration and mode on post-exercise oxygen consumption. Sports Medicine, Auckland, v. 33, n. 14, p. 1037-1060, 2003. Disponível em:

http://www.ncbi.nlm.nih.gov/entrez/query.fcgi?cmd $=$ Retrieve \&db=PubMed\&dopt=Citation\&list uids $=1$ 4599232. Acesso em 10 de janeirofevereiro de 2010

BRANDON, L. J. Physiological factors associated with middle distance running performance. Sports Medicine, Auckland, v. 19, n. 4, p. 268-277, 1995. Disponível em:

http://www.ncbi.nlm.nih.gov/entrez/query.fcgi?cmd $=$ Retrieve\&db=PubMed\&dopt=Citation\&list uids $=7$ 604199. Acesso em 20 de janeiro de 2010

CAPUTO, F.; DENADAI, B. S. Effects of aerobic endurance training status and specificity on oxygen uptake kinetics during maximal exercise.

European Journal of Applied Physiology, Berlin, v. 93, n. 1-2, p. 87-95, 2004. Disponível em:

http://www.ncbi.nlm.nih.gov/entrez/query.fcgi?cmd =Retrieve\&db=PubMed\&dopt=Citation\&list uids $=1$ 5248068. Acesso em 07 de fevereiro de 2010

DE LUCAS, R. D.; DENADAI, B. S.; GRECO, C. C. Respostas fisiológicas durante o exercício contínuo e intermitente: implicações para a avaliação e a prescrição do treinamento aeróbio.

Motriz, Rio Claro, v. 15, n. 4, p. 810-820, 2009. Disponível em:

http://www.periodicos.rc.biblioteca.unesp.br/index. php/motriz/article/view/3098. Acesso em 12 de janeiro de 2010

DEMARIE, S.; KORALSZTEIN, J. P.; BILLAT, V. Time limit and time at VO2max' during a continuous and an intermittent run. The Journal of sports medicine and physical fitness, Torino, v. 40, n. 2, p. 96-102, 2000. Disponível 
em:

http://www.ncbi.nlm.nih.gov/entrez/query.fcgi?cmd $=$ Retrieve\&db=PubMed\&dopt=Citation\&list uids $=1$ 1034428. Acesso em 13 de janeiro de 2010

DUPONT, G.; BERTHOIN, S. Time spent at a high percentage of $\mathrm{VO} 2 \mathrm{max}$ for short intermittent runs: active versus passive recovery. Canadian journal of applied physiology, Champaign, v. 29 Suppl, p. S3-S16, 2004. Disponível em:

http://www.ncbi.nlm.nih.gov/entrez/query.fcgi?cmd $=$ Retrieve \&db=PubMed\&dopt=Citation\&list uids $=1$ $\underline{5602083}$. Acesso em 10 de janeiro de 2010

DUPONT, G.; BLONDEL, N.; LENSEL, G.; BERTHOIN, S. Critical velocity and time spent at a high level of VO2 for short intermittent runs at supramaximal velocities. Canadian journal of applied physiology, Champaign, v. 27, n. 2, p. 103-115, 2002. Disponível em: http://www.ncbi.nlm.nih.gov/entrez/query.fcgi?cmd =Retrieve\&db=PubMed\&dopt=Citation\&list uids $=1$ 2179954. Acesso em 16 de janeiro de 2010

ESSEN, B.; HAGENFELDT, L.; KAIJSER, L. Utilization of blood-borne and intramuscular substrates during continuous and intermittent exercise in man. The Journal of physiology, London, v. 265, n. 2, p. 489-506, 1977. Disponível em:

http://www.ncbi.nlm.nih.gov/entrez/query.fcgi?cmd $=$ Retrieve\&db=PubMed\&dopt=Citation\&list uids $=8$ 50204. Acesso em 23 de março de 2010

FOX, E. L.; BARTELS, R. L.; BILLINGS, C. E.; MATHEWS, D. K.; BASON, R.; WEBB, W. M. Intensity and distance of interval training programs and changes in aerobic power. Medicine and science in sports, Madison, v. 5, n. 1, p. 18-22, 1973. Disponível em:

http://www.ncbi.nlm.nih.gov/entrez/query.fcgi?cmd =Retrieve \&db=PubMed\&dopt $=$ Citation\&list uids $=4$ 721844. Acesso em 24 de janeiro de 2010

GOROSTIAGA, E. M.; WALTER, C. B.; FOSTER, C.; HICKSON, R. C. Uniqueness of interval and continuous training at the same maintained exercise intensity. European Journal of Applied Physiology and Occupational Physiology, Berlin, v. 63, n. 2, p. 101-107, 1991. Disponível em:

http://www.ncbi.nlm.nih.gov/entrez/query.fcgi?cmd $=$ Retrieve \&db=PubMed\&dopt=Citation\&list uids $=1$ 748098. Acesso em 28 de janeiro de 2010

HAWKINS, M. N.; RAVEN, P. B.; SNELL, P. G.; STRAY-GUNDERSEN, J.; LEVINE, B. D. Maximal oxygen uptake as a parametric measure of cardiorespiratory capacity. Medicine and science in sports and exercise, Madison, v. 39, n. 1, p. 103-107, 2007. Disponível em:

http://www.ncbi.nlm.nih.gov/entrez/query.fcgi?cmd
$=$ Retrieve $\& \mathrm{db}=$ PubMed\&dopt=Citation\&list uids $=1$ 7218891. Acesso em 23 de janeiro de 2010

HILL, A. V.; LUPTON, H. Muscle exercise, lactic acid, and the supply and utilization of oxygen. Quarterly Journal of Medicine, Oxford, v. 16, n. 62 , p. 135-171, 1923.

HILL, D. W.; POOLE, D. C.; SMITH, J. C. The relationship between power and the time to achieve . $\mathrm{VO}(2 \mathrm{max})$. Medicine and science in sports and exercise, Madison, v. 34, n. 4, p. 709714, 2002. Disponível em:

http://www.ncbi.nlm.nih.gov/entrez/query.fcgi?cmd $=$ Retrieve \&db=PubMed\&dopt=Citation\&list uids $=1$ 1932583. Acesso em 10 de novembro de 2009

IAIA, F. M.; BANGSBO, J. Speed endurance training is a powerful stimulus for physiological adaptations and performance improvements of athletes. Scandinavian journal of medicine \& science in sports, Copenhagen, v. 20 Suppl 2, p. 11-23, 2010. Disponível em:

http://www.ncbi.nlm.nih.gov/entrez/query.fcgi?cmd $=$ Retrieve\&db=PubMed\&dopt=Citation\&list uids=2 0840558. Acesso em 14 de abril de 2010

KATCH, V. L.; SADY, S. S.; FREEDSON, P. Biological variability in maximum aerobic power. Medicine and science in sports and exercise, Madison, v. 14, n. 1, p. 21-25, 1982. Disponível em:

http://www.ncbi.nlm.nih.gov/entrez/query.fcgi?cmd $=$ Retrieve\&db=PubMed\&dopt=Citation\&list uids $=7$ 070252. Acesso em 12 de março de 2010

MACDOUGALL, J. D.; HICKS, A. L.; MACDONALD, J. R.; MCKELVIE, R. S.; GREEN, H. J.; SMITH, K. M. Muscle performance and enzymatic adaptations to sprint interval training. Journal of Applied Physiology, Bethesda, v. 84, n. 6, p. 2138-2142, 1998. Disponível em:

http://www.ncbi.nlm.nih.gov/entrez/query.fcgi?cmd $=$ Retrieve \&db=PubMed\&dopt=Citation\&list uids $=9$ 609810. Acesso em 10 de janeiro de 2010

MIDGLEY, A. W.; MC NAUGHTON, L. R. Time at or near VO2max during continuous and intermittent running. A review with special reference to considerations for the optimisation of training protocols to elicit the longest time at or near VO2max. The Journal of sports medicine and physical fitness, Torino, v. 46, n. 1, p. 1-14, 2006. Disponível em:

http://www.ncbi.nlm.nih.gov/entrez/query.fcgi?cmd =Retrieve\&db=PubMed\&dopt=Citation\&list uids $=1$ 6596093. Acesso em 21 de janeiro de 2010

MIDGLEY, A. W.; MCNAUGHTON, L. R.; CARROLL, S. Time at VO2max during intermittent treadmill running: test protocol dependent or methodological artefact? International journal of sports medicine, Stuttgart, v. 28, n. 11, p. 934- 
939, 2007. Disponível em:

http://www.ncbi.nlm.nih.gov/entrez/query.fcgi?cmd $=$ Retrieve\&db=PubMed\&dopt=Citation\&list uids $=1$ 7497578. Acesso em 20 de janeiro de 2010

MIDGLEY, A. W.; MCNAUGHTON, L. R.; JONES, A. M. Training to enhance the physiological determinants of long-distance running performance: can valid recommendations be given to runners and coaches based on current scientific knowledge? Sports Medicine, Auckland, v. 37, n. 10, p. 857-880, 2007. Disponível em:

http://www.ncbi.nlm.nih.gov/entrez/query.fcgi?cmd $=$ Retrieve\&db=PubMed\&dopt=Citation\&list uids $=1$ 7887811. Acesso em 23 de janeiro de 2010

MIDGLEY, A. W.; MCNAUGHTON, L. R.; WILKINSON, $M$. Is there an optimal training intensity for enhancing the maximal oxygen uptake of distance runners?: empirical research findings, current opinions, physiological rationale and practical recommendations. Sports

Medicine, Auckland, v. 36, n. 2, p. 117-132, 2006. Disponível em:

http://www.ncbi.nlm.nih.gov/entrez/query.fcgi?cmd =Retrieve\&db=PubMed\&dopt=Citation\&list uids $=1$ $\underline{6464121}$. Acesso em 12 de janeiro de 2010

MILLET, G. P.; CANDAU, R.; FATTORI, P.; BIGNET, F.; VARRAY, A. VO2 responses to different intermittent runs at velocity associated with VO2max. Canadian journal of applied physiology, Champaign, v. 28, n. 3, p. 410-423, 2003. Disponível em:

http://www.ncbi.nlm.nih.gov/entrez/query.fcgi?cmd $=$ Retrieve \&db=PubMed\&dopt=Citation\&list uids $=1$ 2955868. Acesso em 05 de fevereiro de 2010

MILLET, G. P.; LIBICZ, S.; BORRANI, F.; FATTORI, P.; BIGNET, F.; CANDAU, R. Effects of increased intensity of intermittent training in runners with differing VO2 kinetics. European Journal of Applied Physiology, Berlin, v. 90, n. 1-2, p. 50-57, 2003. Disponível em: http://www.ncbi.nlm.nih.gov/entrez/query.fcgi?cmd $=$ Retrieve\&db=PubMed\&dopt=Citation\&list uids $=1$ 2811566. Acesso em 03 de fevereiro de 2010

NOAKES, T. D.; MYBURGH, K. H.; SCHALL, R. Peak treadmill running velocity during the $\mathrm{VO} 2$ max test predicts running performance. Journal of sports sciences, London, v. 8, n. 1, p. 35-45, 1990. Disponível em:

http://www.ncbi.nlm.nih.gov/entrez/query.fcgi?cmd $=$ Retrieve \&db=PubMed\&dopt=Citation\&list uids $=2$ 359150. Acesso em 04 de fevereiro de 2010

ROBINSON, D. M.; ROBINSON, S. M.; HUME, P. A.; HOPKINS, W. G. Training intensity of elite male distance runners. Medicine and science in sports and exercise, Madison, v. 23, n. 9, p. 1078-1082, 1991. Disponível em: http://www.ncbi.nlm.nih.gov/entrez/query.fcgi?cmd $=$ Retrieve\&db=PubMed\&dopt $=$ Citation\&list uids $=1$ 943629. Acesso em 10 de março de 2010

ROZENEK, R.; FUNATO, K.; KUBO, J.; HOSHIKAWA, M.; MATSUO, A. Physiological responses to interval training sessions at velocities associated with VO2max. Journal of strength and conditioning research, Champaign, v. 21, n. 1, p. 188-192, 2007. Disponível em:

http://www.ncbi.nlm.nih.gov/entrez/query.fcgi?cmd $=$ Retrieve \&db=PubMed\&dopt $=$ Citation\&list uids $=1$ 7313282. Acesso em 01 de março de 2010

SPRIET, L. L.; HOWLETT, R. A.; HEIGENHAUSER, G. J. An enzymatic approach to lactate production in human skeletal muscle during exercise. Medicine and science in sports and exercise, Madison, v. 32, n. 4, p. 756-763, 2000. Disponível em:

http://www.ncbi.nlm.nih.gov/entrez/query.fcgi?cmd $=$ Retrieve \&db=PubMed\&dopt=Citation\&list uids $=1$ 0776894. Acesso em 10 de abril de 2010

THEVENET, D.; LECLAIR, E.; TARDIEUBERGER, M.; BERTHOIN, S.; REGUEME, S.; PRIOUX, J. Influence of recovery intensity on time spent at maximal oxygen uptake during an intermittent session in young, endurance-trained athletes. Journal of sports sciences, London, $v$. 26, n. 12, p. 1313-1321, 2008. Disponível em: http://www.ncbi.nlm.nih.gov/entrez/query.fcgi?cmd $=$ Retrieve \&db=PubMed\&dopt=Citation\&list uids $=1$ 8821267. Acesso em 10 de março de 2010

THEVENET, D.; TARDIEU, M.; ZOUHAL, H.; JACOB, C.; ABDERRAHMAN, B. A.; PRIOUX, J. Influence of exercise intensity on time spent at high percentage of maximal oxygen uptake during an intermittent session in young endurancetrained athletes. European Journal of Applied Physiology, Berlin, v. 102, n. 1, p. 19-26, 2007. Disponível em:

http://www.ncbi.nlm.nih.gov/entrez/query.fcgi?cmd $=$ Retrieve \&db=PubMed\&dopt=Citation\&list uids $=1$ 7851682. Acesso em 15 de janeiro de 2010

TURNER, A. P.; CATHCART, A. J.; PARKER, M. E.; BUTTERWORTH, C.; WILSON, J.; WARD, S. A. Oxygen uptake and muscle desaturation kinetics during intermittent cycling. Medicine and science in sports and exercise, Madison, v. 38, n. 3, p. 492-503, 2006. Disponível em:

http://www.ncbi.nlm.nih.gov/entrez/query.fcgi?cmd =Retrieve\&db=PubMed\&dopt=Citation\&list uids $=1$ $\underline{6540837}$. Acesso em 13 de janeiro de 2010

WENGER, H. A.; BELL, G. J. The interactions of intensity, frequency and duration of exercise training in altering cardiorespiratory fitness.

Sports Medicine, Auckland, v. 3, n. 5, p. 346356, 1986. Disponível em: 
http://www.ncbi.nlm.nih.gov/entrez/query.fcgi?cmd

$=$ Retrieve\&db=PubMed\&dopt=Citation\&list uids $=3$ 529283. Acesso em 09 de fevereiro de 2010

\section{Endereço:}

Fabrizio Caputo

Rua Pascoal Simone, 358 Bairro: Coqueiros

Florianópolis SC Brasil

88080-350

Telefone: (48) 3321-8641

Fax: (48) 3321-8607

e-mail: fabriziocaputo@hotmail.com

Recebido em: 13 de outubro de 2011.

Aceito em: 19 de fevereiro de 2013.

\section{(c) (i)}

Motriz. Revista de Educacão Física. UNESP, Rio Claro,

SP, Brasil - elSSN: 1980-6574 - está licenciada sob

Creative Commons - Atribuicão 3.0 